\title{
Frequency Response Analysis of a Nonstationary System: Electrochemical Transition of Polyaniline
}

\author{
Katja Magdić Košiček, * Višnja Horvat-Radošević, Krešimir Kvastek
}

\author{
Division for Marine and Environmental Research, Ruđer Bošković Institute, Bijenička c. 54, HR-10000 Zagreb, Croatia \\ * Corresponding author's e-mail address: kmagdic@irb.hr
}

RECEIVED: November 28, 2018 * REVISED: January 24, 2019 * ACCEPTED: January 24, 2019

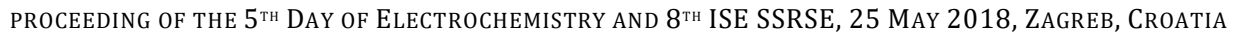

\begin{abstract}
In this work, impedance spectra of polyaniline film electrode recorded at some redox transition potentials have been corrected for nonstationarity by application of Stoynov's 4-D method. The procedure consisted from: a) conventional measurements of a series of impedance spectra under strictly same experimental conditions, which are accompanied by monitoring of real operating time intervals for measurement of each particular impedance spectrum of the series, b) determination of functional dependence(s) between iso-frequency impedance values and operating time, and c) calculations of instantaneous IS for the time instant of the beginning of the first IS measurement. Comparison between measured and instantaneous impedance spectra, and also between their individual impedance parameter values pointed toward significant differences in charge transfer and transport resistance values. The results suggest that erroneous (either underestimated or overestimated) charge transfer and transport resistance values will be obtained if measured impedance spectra were not corrected for nonstationarity.
\end{abstract}

Keywords: nonstationary impedance spectra, instantaneous impedance spectra, polyaniline film electrode, redox transition states.

\section{INTRODUCTION}

$\mathbf{E}$ LECTROCHEMICAL impedance spectroscopy (EIS) ${ }^{[1,2]}$ is the extremely powerful technique for characterization of either different electrode materials toward their electrical/dielectrical, ${ }^{[3]}$ electrocatalytic ${ }^{[4]}$ and corrosive properties, ${ }^{[5]}$ or different electrochemical devices such as energy conversion/storage devices ${ }^{[6]}$ or electrochemical sensors. ${ }^{[7]}$ Impedance measurements are based on measuring the electrical response to some small periodic electrical excitation of the system. More specifically, impedance is defined as the quotient between Fourier transforms of differential equations defining the sinusoidal voltage response and sinusoidal current excitation. Only under conditions of linearity, causality and stationarity, the quotient would be reduced to the simple Ohm's law by which the complex and frequency dependent resistance, i.e. impedance, becomes defined..$^{[1,2]}$ Violation from any of these conditions prevents all possibly measured impedance spectra to be true impedance spectra prone to accurate impedance data analysis.
The most common violation is related to the condition of stationarity, because many electrochemical systems involve nonsteady-state processes that cause permanent system changes such as corrosion, ${ }^{[8]}$ deposition, ${ }^{[9]}$ or system poisoning. ${ }^{[10]}$ Conventional impedance spectra measurements by frequency response analysis (FRA) are generally performed at a succession of different frequencies. Thus, in dependence on the frequency range, number of frequencies per decade and integration time explored, the single impedance spectrum measurement can last for hours. ${ }^{[11,12]} \mathrm{A}$ critical situation emerges when a measured system is changing between the beginning and the end of the single impedance spectrum measurement when impedance data couldn't be analysed in terms of steady-state models. ${ }^{[13]}$

In order to eliminate nonstationarity, the finding of a method for determination of instantaneous impedance spectra, i.e. impedance spectra defined at one strictly defined time instant for all frequencies, has already been detected as crucial for accurate impedance data analysis. ${ }^{[14-18]}$ Heretofore, instantaneous impedance 
spectra can be obtained by two principal methods. The first method is concerned to impedance measurements by dynamic electrochemical impedance spectroscopy (DEIS) technique. ${ }^{[15,18]}$ DEIS is based on application of multi sinusoidal signals of different frequencies, short Fourier transformations and consequent impedance data calculations. Analyses of instantaneous impedance spectra obtained by DEIS have already been reported for various time changing systems such corrosion systems, ${ }^{[8]}$ fuel cell under operation conditions ${ }^{[19]}$ and some other electrocatalytic reactions. ${ }^{[20]}$ The second method is based on $a$ posteriori corrections of impedance spectra measured by the conventional EIS and subsequent calculations of instantaneous impedance spectra on the basis of the so called four-dimensional (4-D) analysis. ${ }^{[14,21]} 4-D$ analysis has to be performed using a number of sequentially measured impedance spectra and monitoring the operating time of measurements as an additional experimental variable. By defining an adequate mathematical function between isofrequency impedance values and operating time, instantaneous impedance spectra can be calculated for any desired time by the extrapolation/interpolation method. Although being claimed as an approximate method, ${ }^{[22]} 4-D$ analysis has been successfully applied to time changing impedance spectra of some conducting polymers. ${ }^{[23-26]}$

Conducting polymers belong to a special class of novel materials with broad applications due to various beneficial properties, among which a tunable conductivity induced between insulating and conducting polymer states is the most significant. ${ }^{[27-29]}$ Tunable conductivity of conducting polymers is usually insured by electrochemical switching, i.e. electrochemical oxidation/reduction coupled by insertion/deinsertion of solution ions (protons and/or counterions). Due to the complex structure of polymer, this process can be rather slow and cause long time changes of the system ${ }^{[29-31]}$. Anyway, the conventional EIS technique has frequently been applied for investigations of charge storage, transfer and transport properties of conducting polymers. ${ }^{[32-34]}$ Moreover, the conventional EIS technique has already been applied for investigation of time changing of polyaniline (PANI) in its partially reduced states. ${ }^{[35,36]}$. Short EIS measurements were performed, assuming that time change of PANI is slower than duration of the single spectrum measurement. Comparison of conventionally measured and instantaneous impedance spectra obtained by 4-D analysis for poly(o-ethoxyaniline) (POEA) submitted to a partial electrochemical reduction ${ }^{[23]}$ and overoxidized poly(3,4-ethylenedioxythiophene) (PEDOT) aged at the reducing potential,[24-26] however, showed significant differences. Since these differences would generate differences in impedance parameter values that are crucial for proper appreciation and assessment of all underlying processes, the correction procedure of EIS data obtained for nonstationary systems becomes of outmost importance.

The main objective of the present work is to perform 4-D analysis of nonstationary impedance spectra recorded for PANI film in the sulphuric acid medium at some redox transition potentials and to construct instantaneous impedance spectra. The intent would also be to compare conventionally measured and instantaneous impedance spectra and to find which between impedance parameters obtained by the conventional EIS technique would be mostly affected by nonstationarity.

\section{EXPERIMENTAL}

\section{Polyaniline (PANI) Film Preparation}

PANI film was synthesized by oxidative polymerization of aniline on the Pt-wire electrode $\left(0.088 \mathrm{~cm}^{2}\right)$ serving as the working electrode in a three-electrode glass cell. Pt-coil served as the counter, and the saturated calomel electrode (SCE), equipped with the Luggin capillary and pseudoreference Pt-wire, ${ }^{[37]}$ served as the reference electrode. Three-electrode cell was thermostated at $25^{\circ} \mathrm{C}$. Solartron Analytical $1287 \mathrm{ECl}$ under Zcorr software (Scribner Assoc. Inc.) was used for all voltammetric measurements. The synthesis solution was $0.1 \mathrm{~mol} \mathrm{dm}^{-3}$ aniline (Merck) in 0.5 mol dm ${ }^{-3} \mathrm{H}_{2} \mathrm{SO}_{4}$ (Kemika 96\%) aqueous (Milli-Q) solution, which was deoxygenated with high purity $\mathrm{N}_{2}$ bubbling before the polymerization experiment. The monomer was used as received. Similarly to the procedure described before, ${ }^{[35]}$ oxidative polymerization was performed by cycling the potential at a sweep rate, $v=50 \mathrm{mV} \mathrm{s}^{-1}$, between the negative limit at $-0.20 \mathrm{~V} v$ s. SCE and positive limit at $1.00 \mathrm{~V}$ vs. SCE in the $1^{\text {st }}$ cycle (corresponding the beginning of aniline oxidation) and at $0.85 \mathrm{~V}$ vs. SCE in all subsequent 24 potential cycles (Figure 1a). Two prominent voltammetric peaks in Figure 1a are characteristic for different oxidation states of PANI and associated with different levels of conductivity. ${ }^{[27-30]}$ The first peak at $0.15 \mathrm{~V}$ vs. SCE corresponds to the leucoemeraldine (LE)/emeraldine (EM), while the second peak at $0.75 \mathrm{~V} v s$. SCE is due to the emeraldine (EM)/pernigraniline (PE) redox transitions. The potential region between the first (LE-EM) and the second (EM-PE) peak is the range where PANI manifests the maximum conductivity of the PANI-EM state. ${ }^{[27-30]}$ The procedure of cyclization was stopped at $0.85 \mathrm{~V}$ vs. SCE, and PANI coated electrode was pulled out, rinsed, transferred to deaerated and aniline free $0.05 \mathrm{~mol}$ $\mathrm{dm}^{-3} \mathrm{H}_{2} \mathrm{SO}_{4}$ solution, and polarized at $0.45 \mathrm{~V}$ vs. SCE. The electrode is than cycled at the sweep rate of $50 \mathrm{mV} \mathrm{s}^{-1}$ between $0.45 \mathrm{~V}$ and $-0.20 \mathrm{~V}$ vs. SCE, covering EM-LE and LEEM transitions, until the stationary current response was achieved. Two first cycles are shown in Figure 1b. The 
a

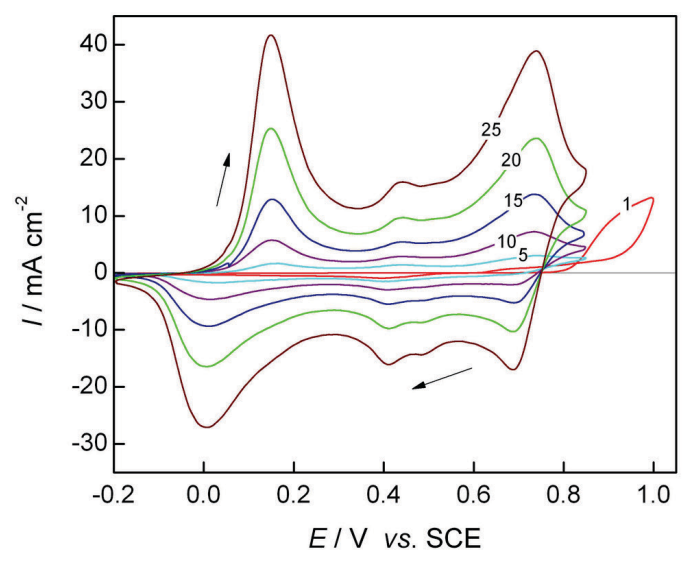

b

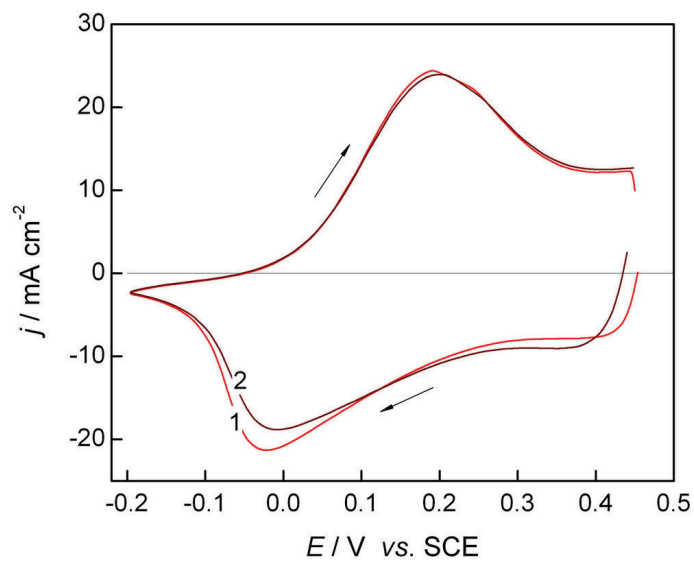

Figure 1. Cyclic voltammograms at $v=50 \mathrm{mV} \mathrm{s} \mathrm{s}^{-1}$ of: a) $1^{\text {st }}, 5^{\text {th }}, 10^{\text {th }}, 15^{\text {th }}, 20^{\text {th }}$ and $25^{\text {th }}$ cycle of aniline electro-polymerization from $0.1 \mathrm{~mol} \mathrm{dm}^{-3}$ aniline $/ 0.5 \mathrm{~mol} \mathrm{dm}^{-3} \mathrm{H}_{2} \mathrm{SO}_{4}$ and b) PANI film in $0.05 \mathrm{~mol} \mathrm{dm}^{-3} \mathrm{H}_{2} \mathrm{SO}_{4}$ (1 ${ }^{\text {st }}$ and $2^{\text {nd }} \mathrm{cycle}$ ).

voltammetric deposition charge of $140 \mathrm{mC} \mathrm{cm}^{-2}$, serving as a measure of the film thickness, was calculated through integration of the anodic part of CV presented in Figure $1 \mathrm{~b}$.

\section{Polarization Treatment of PANI Film}

Prior impedance measurements, PANI film was held overnight at $-0.20 \mathrm{~V}$ vs. SCE to form the insulating, PANI-LE state. For insulating to conducting (LE-EM) and reversed (EM-LE) transitions, polarization potential values were stepped in $50 \mathrm{mV}$ from $-0.20 \mathrm{~V}$ (reduced, LE state) to 0.45 $\mathrm{V}$ (half-oxidized, EM state) and inversely, from $0.45 \mathrm{~V}$ to $0.20 \mathrm{~V}$ vs. SCE, what is similar to the already reported procedure ${ }^{[30]}$. Polarization potentials were held at each value for 5 minutes, prior at least two consecutive impedance spectra measurements were performed. If changes in impedance spectra were recorded, measurements would be repeated until stationarity was attained. After that, the potential was stepped (either positively or negatively) to another potential value and the whole procedure of impedance spectra measurements was repeated. All experiments were performed with the same PANI film in the thermostated $\left(25^{\circ} \mathrm{C}\right)$ three-electrode cell described above.

\section{Impedance Spectra Measurements}

Impedance spectra (IS) measurements of PANI electrode were carried out using the Solartron Analytical equipment (1287 ECl and 1260 FRA) under Zplot (Scribner Assoc. Inc.) software control. IS measurements were performed over the frequency $(f)$ range between $1 \times 10^{5}$ and $0.10 \mathrm{~Hz}$. Prior measurements, the amplitude of the potential perturbation of $0.01 \mathrm{~V}$, integration time $\left(t_{\text {in }}\right)$ of $10 \mathrm{~s}$, and time delay before each frequency point measurement $\left(t_{\mathrm{d}}\right)$ of $1 \mathrm{~s}$ were pre-selected. IS were measured at ten frequencies per decade, resulting in 60 measured frequency points for the single IS. The time schedule of the single IS measurement in given conditions is presented in Figure 2, where $t_{\mathrm{s}}$ and $t_{\mathrm{e}}$ denote characteristic time instants of the start and the end of IS measurement, while $t_{\mathrm{c}}{ }^{(\mathrm{k})}$ denotes the central time instant of measurement at each frequency point $f^{(\mathrm{k})}(\mathrm{k}=1-60)$.

It is obvious from Figure 2 that the total time for single IS measurement is defined as $t_{\mathrm{m}}=t_{\mathrm{e}}-t_{\mathrm{s}}$. Whereas $t_{\mathrm{m}}$ depends primarily on the frequency range and number of explored frequencies per decade, time needed for each frequency measurement should always be the same and equal to the pre-selected value of $t_{\text {in }}$. Here, $t_{m}$ was

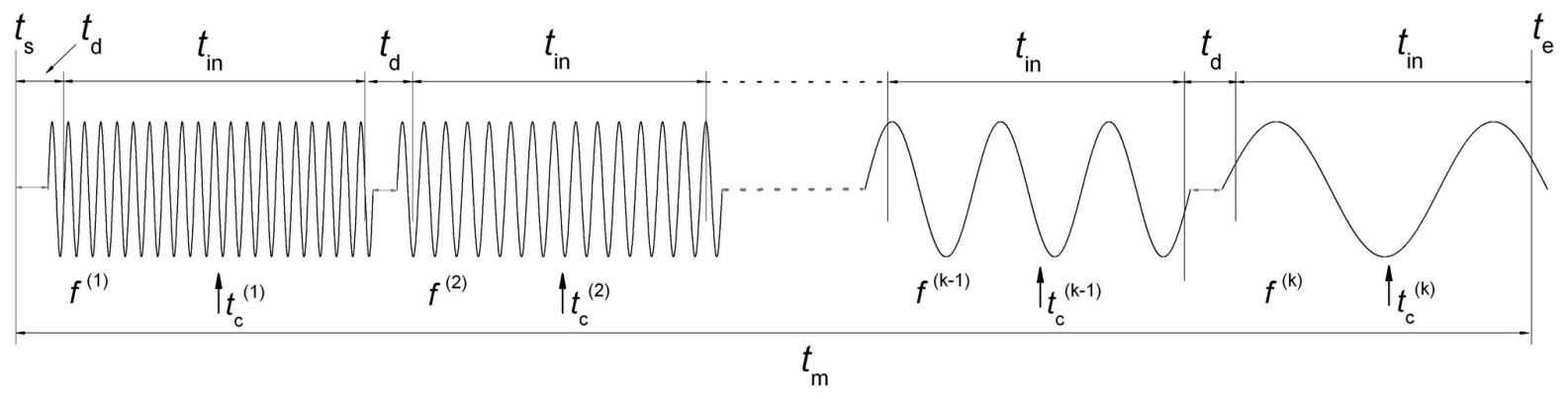

Figure 2. Schematic time schedule of single IS measurement. 


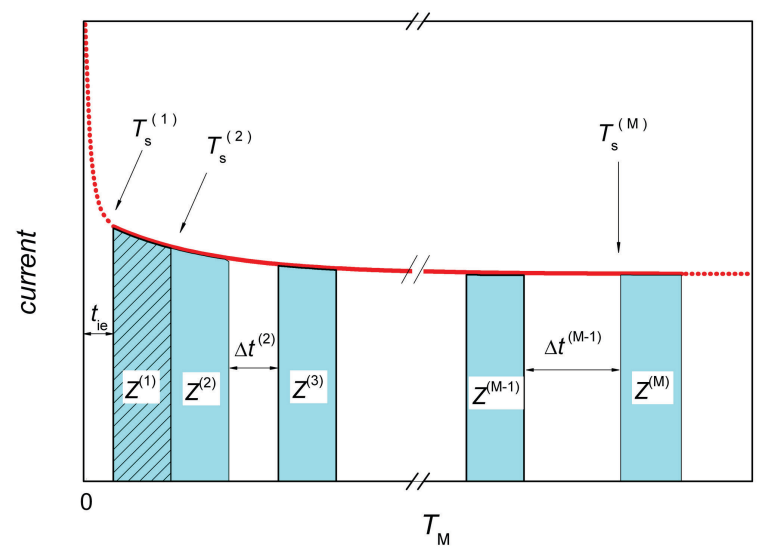

Figure 3. Schematic time schedule of a series of sequentially measured IS.

determined as $725 \mathrm{~s}$, what suggests that time of measurements at low frequencies was somewhat longer than $t_{\text {in. }}{ }^{[23]}$ Each single IS measurement resulted in the usual experimental 3-D set, containing a pair of impedance values $\left(Z^{\prime(k)}, Z^{\prime \prime}(k)\right)$ or $\left(\log |Z|^{(k)}, \varphi^{(k)}\right)$ for each measured frequency $\left(f^{(k)}=\omega^{(k)} / 2 \pi\right)$, where $\omega$ is radial frequency. Note that $Z^{\prime}, Z^{\prime \prime},|Z|$ and $\varphi$ are real impedance part, imaginary impedance part, impedance modulus and phase angle, respectively.

At potential values where nonstationary impedance spectra were observed, a number (M) of consecutively measured IS (from $Z^{(1)}$ to $Z^{(M)}$ ) were performed according to the time schedule presented in Figure 3. All consecutive IS were measured at strictly same conditions (polarization potential, perturbation signal amplitude, frequency range, number of frequency points per decade, $t_{\text {in }}$ and $\left.t_{\mathrm{d}}\right)$. The operating time $\left(T_{\mathrm{M}}\right)$ of the whole experiment was measured from the time instant of PANI electrode polarization and includes 5 minutes of the initial system equilibration $\left(t_{\text {ie }}\right)$. In Figure 3 , specific times of the beginning of each IS measurement $\left(T_{s}^{(\mathrm{M})}\right)$ and time delay $\left(\Delta t^{(\mathrm{M})}\right)$ between IS measurements are labelled. The total number (M) of consecutively measured IS was dependent on the rate by which steady-state (or equal IS) was obtained.

It is obvious from Figure 3 that each IS (from $Z^{(1)}$ to $\left.Z^{(\mathrm{M})}\right)$ is actually measured at different operating time $\left(T_{\mathrm{M}}\right)$ intervals, what implies that characteristic $t_{c}{ }^{(k)}$ values which are for every single IS referred to the corresponding $T_{s}(\mathrm{M})$ (cf. Figure 2), become also different. Here, all $t_{c}^{(k)}$ values referred to $T_{\mathrm{M}}=0$ are denoted as $t_{\mathrm{cM}}{ }^{(k)}=T_{s}^{(\mathrm{M})}+t_{c}^{(k)}$. By monitoring $T_{\mathrm{M}}$ and calculating $t_{\mathrm{cM}}{ }^{(k)}$ as the most characteristic time instant, ${ }^{[23]}$ the whole experiment performed at some specific polarization potential resulted in a series of $M$ impedance 4-D sets of either $\left(Z^{\prime}(k), Z^{\prime \prime}(k), f^{(k)}\right.$, $\left.t_{\mathrm{cM}}^{(\mathrm{k})}\right)$ or $\left(\log |Z|^{(\mathrm{k})}, \varphi^{(\mathrm{k})}, f^{(\mathrm{k})}, t_{\mathrm{cM}}^{(\mathrm{k})}\right)$.

\section{Calculations and Fittings}

Complex non-linear least square (CNLS) fitting code was applied by the Zview (Scribner Assoc. Inc.) software for impedance spectra analysis. Non-linear least square analysis (NLS) was applied by using the Origin (OriginLab Corp.) software for fitting iso-frequency impedance vs. time curves, determination of curve parameters and interpolating procedure. All fittings were performed on the basis of acceptable $\chi^{2}$ tests and small relative standard deviations $(r s d)$ of parameter values.

\section{RESULTS AND DISCUSSION}

\section{Experimental Impedance Spectra}

Experimentally obtained impedance 3-D sets for two consecutively measured IS at specified potential values reached in either positive or negative directions of polarization potential changes, are in the form of Bode plots $(\log |Z|$ and $\varphi$ vs. $\log \omega)$ presented in Figure 4.

Impedance values and types of impedance/frequency responses at different polarization potentials are in general agreement to those already obtained for PANI films at various oxidation states. ${ }^{[9,35-45]}$ Impedance due to experimental artefacts ${ }^{[3,37]}$ and pure resistive impedance response which is mainly due to the uncompensated solution resistance, appeared at the highest frequencies, and are followed by PANI film impedance response at lower frequencies ${ }^{[32-34]}$. As expected, the impedance magnitude of PANI film is the highest for the reduced, PANI-LE state at $-0.20 \mathrm{~V}$ and the lowest for the half-oxidized, PANI-EM state at $0.45 \mathrm{~V}$ vs. SCE. At this last potential value, almost purely capacitive phase angle response $\left(c a .-90^{\circ}\right)$ is due to the pseudocapacitance of the interior of the open structure of the conducting PANI-EM form ${ }^{[38-43]}$. Note that PANI film pseudocapacitance is generated by fast redox reaction of polymer and responsible for respective charge storage properties of PANI-EM form. ${ }^{[28,42,43]} \mathrm{At}-0.20 \mathrm{~V}$ vs. SCE, highly resistive PANI film having a closed structure of inactive PANI-LE form is, in dependence on PANI "history" before measurements, [29-31] indicated by either pure double-layer capacitive phase angle (ca. $-90^{\circ}$ ) response (Figure $\left.4 a\right)$, or combined with a Warburglike phase angle $\left(c a .-45^{\circ}\right)$ response generated by slow charge transport within the PANI film ${ }^{[38,44]}$ (Figure $4 \mathrm{~b}$ ). It is important to notice here that IS measured at these two limited potential values do not change considerably with time spent for two consecutive measurements, except for the just reduced PANI. ${ }^{[31,36]}$ This suggests that almost stationary, i.e. quasy-equilibrium states were reached for the fully reduced and relaxed PANI-LE and half-oxidized PANI-EM forms. Therefore, all evaluated impedance parameter values (double-layer capacitance or film pseudo-capacitance) for these two PANI forms would have reliable values. 
a
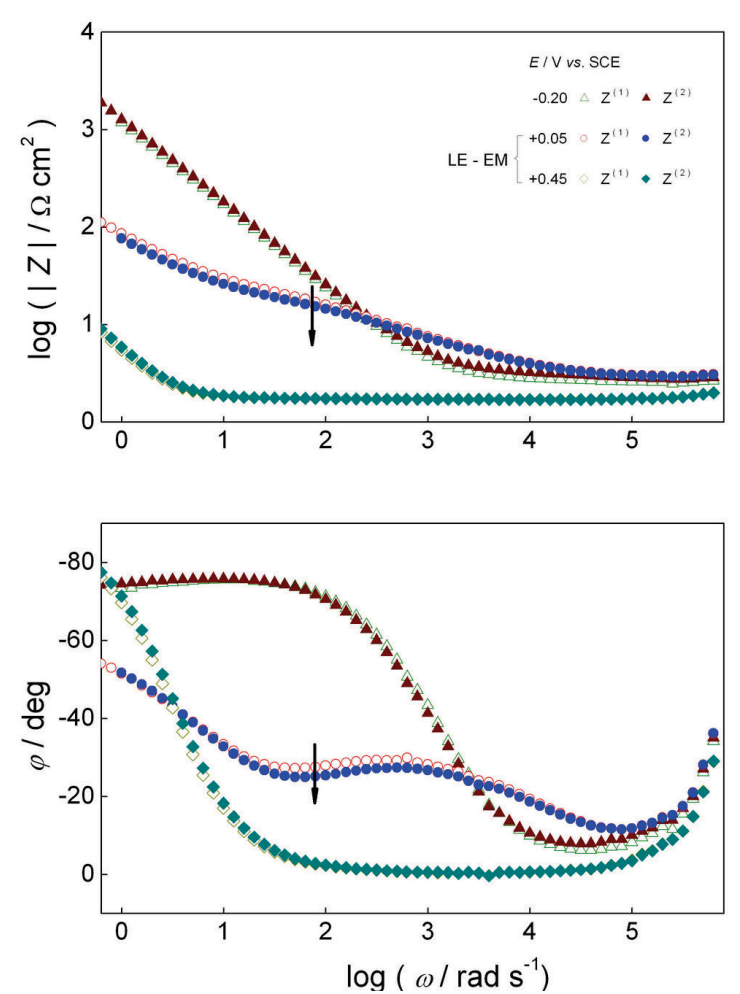

b
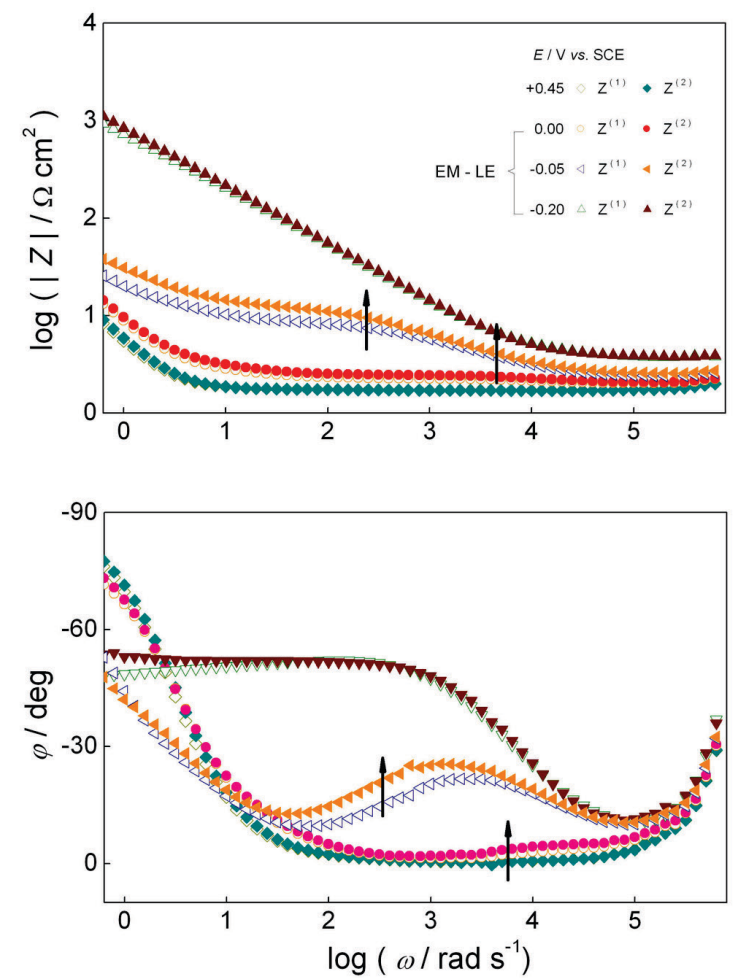

Figure 4. Bode plots of two consecutively measured IS $\left(Z^{(1)}\right.$ and $\left.Z^{(2)}\right)$ of PANI film in $0.05 \mathrm{~mol} \mathrm{dm}^{-3} \mathrm{H}_{2} \mathrm{SO}_{4}$ at denoted polarization potentials reached in a direction of: a) LE-EM redox transition; b) EM-LE redox transition.

IS measured for redox transition states of PANI film induced in both directions of potential changes are, however, more liable to time changes. Although measured at similar polarization potential values, higher impedances and less prominent time changes were observed in the course of LE-EM than EM-LE transition. Differences observed in IS at similar redox transition potential values attained in different directions of potential changes are in full agreement with the hysteresis of CVs, i.e. the shift between redox peaks appearing in Figure 1b. The phenomenon of hysteresis in CVs of PANI films has usually been ascribed to a relatively long relaxation process due to extensive morphological, structural and/or conformational changes of the polymer during redox transition from insulating to conducting states and vice versa. ${ }^{[29,30]}$ Directions of time changes are denoted by arrows in Figure 4, showing decreased impedance modulus for LE-EM transition (Figure 4a) and increased impedance modulus for EM-LE transition (Figure 4b), respectively. It can be noticed from phase angle responses that PANI transitions are accompanied by clear contributions of some resistive impedance response (phase angle $0^{\circ}$ ), what suggests presence of an interfacial charge (electrons and ions) transfer process. ${ }^{[31-33]}$ Contributions of charge transfer resistance are in Figure 4 seen as decrease of phase angle(s) at high to medium frequencies. At medium to low frequencies, phase angles increased again toward ca. $-45^{\circ}$ in Figure $4 \mathrm{a}$, or $c a .-90^{\circ}$ in Figure $4 \mathrm{~b}$. This suggests more prominent contribution of the Warburg-like impedance due to the ion transport within the PANI film in the first case, and less Warburg contribution followed by pseudocapacitive impedance of the PANI film in the second case, respectively. Both is in accordance with a view of a compact PANI structure that should be opened to ion transport during LE-EM transition and an opened PANI structure where ion transport should be hindered in the course of EM-LE transition. ${ }^{[30]}$ Time changes of IS in Figure 4 indicate nonstationarity, implying that all possibly evaluated impedance parameter values (double-layer capacitance, charge transfer and transport resistances and film pseudocapacitance) for PANI film in all redox transition states would be unreliable.

For obtaining reliable impedance parameter values, a number of IS $\left(Z^{(1)}\right.$ to $\left.Z^{(\mathrm{M})}\right)$ were measured according to the time schedule drawn in Figure 3. The results are presented as Nyquist ( $Z^{\prime \prime}$ vs. $Z^{\prime}$ ) plots in Figure 5, where the starting time of each between M IS measurements $\left(T_{s}{ }^{(M)}\right)$ recorded on the $T_{\mathrm{M}}$ scale was taken as the experimental variable. The 
a

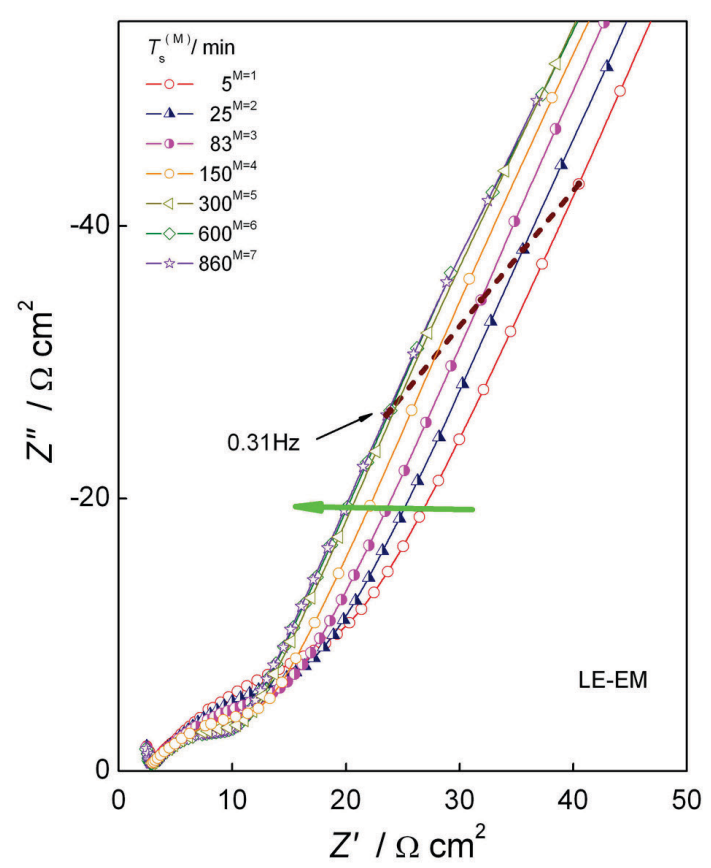

b

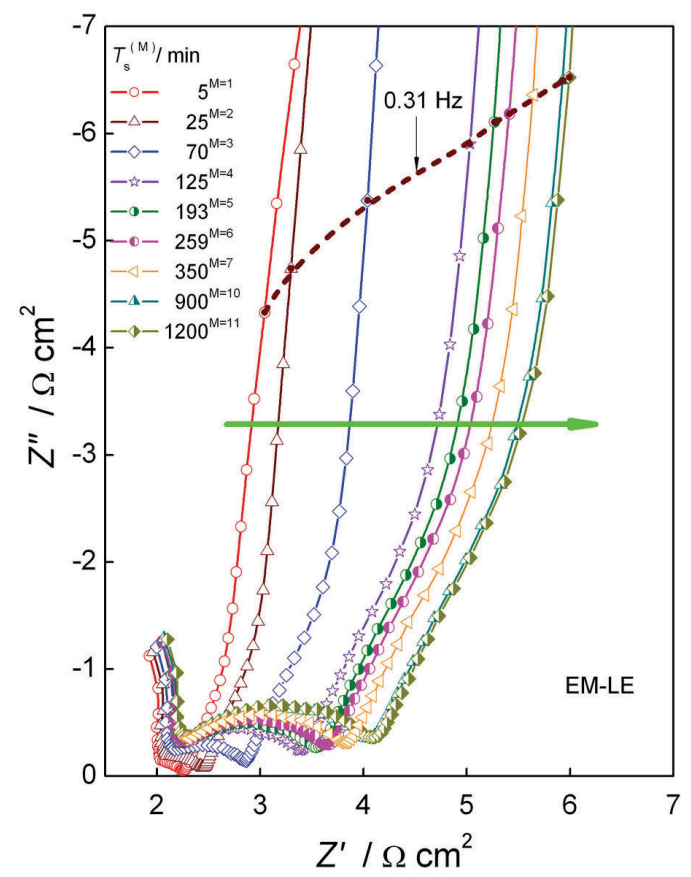

Figure 5. Nyquist plots of sequentially measured IS $\left(Z^{(1)}\right.$ to $\left.Z^{(\mathrm{M})}\right)$ of PANI film in $0.05 \mathrm{~mol} \mathrm{dm}^{-3} \mathrm{H}_{2} \mathrm{SO}_{4}$ at: a) $0.05 \mathrm{~V}$ of LE-EM transition; b) $0.00 \mathrm{~V}$ of EM-LE transition. Impedances at $0.31 \mathrm{~Hz}$ are connected by dotted lines.

series of IS measured at $0.05 \mathrm{~V}$ vs. SCE of LE-EM transition is shown in Figure $5 \mathrm{a}$, while that measured at $0.00 \mathrm{~V}$ vs. SCE of EM-LE transition is shown in Figure $5 b$.

For all Nyquist plots shown in Figure 5, impedances due to appearance of experimental artefacts at the highest frequencies ${ }^{[35,37]}$ are followed by near semicircle responses at high to medium frequencies and more or less vertical lines at lower frequencies. Just the Nyquist type of PANI film impedance representation has been most frequently analysed in the literature. ${ }^{[35-45]}$ Semicircle responses have usually been ascribed to double-layer interfacial capacitance coupled with charge transfer kinetics, while more or less vertical lines are due to more or less overlapped Warburg and pseudo-capacitive impedance responses. Time changes of IS are in Figure 5 denoted by arrows, suggesting either continuous decrease of circle diameters for LE-EM transition (Figure 5a), or continuous increase of circle diameters for EM-LE transition (Figure $5 b$ ). In both cases, impedance vector magnitudes at equal frequency points (marked for $f=0.31 \mathrm{~Hz}$ ) describe almost linear shifts, particularly at longer times, what has already been observed for PANI film aged at a reduction potential. ${ }^{[36]}$

\section{Instantaneous Impedance Spectra Construction}

For instantaneous impedance spectra construction, 4-D set of data was collected for every of consecutively measured
IS $\left(Z^{(1)}\right.$ to $\left.Z^{(\mathrm{M})}\right)$. Impedance data $\left(Z^{\prime}(\mathrm{k}), Z^{\prime \prime}(\mathrm{k})\right)$ of each 4-D set were for every $f^{(k)}(k=1-60)$ presented as a function of the operation time $\left(T_{\mathrm{M}}\right)$ by using the corresponding $T_{s}{ }^{(\mathrm{M})}$ and $t_{\mathrm{CM}}{ }^{(\mathrm{k})}$ values, respectively. The example is presented in Figure 6 , showing three-dimensional plots of measured $Z^{(1)}$ to $Z^{(11)}$ (already presented in Figure $5 b$ ).

The points of equal frequencies (iso-frequency points) at 0.31 and $0.10 \mathrm{~Hz}$ are connected by dotted lines, showing clearly an increase of measured impedance vectors by time elapsed during the whole experiment. Similar has already been noticed not only for IS of PANI, ${ }^{[36]}$ but also for POEA and PEDOT polymer film electrodes at reduction conditions. ${ }^{[23-26]}$

In the following step of instantaneous IS calculation, $Z^{\prime}$ and $Z^{\prime \prime}$ values of each $Z^{(M)}$ where extracted for every frequency $f^{(k)}(k=1-60)$ and drawn separately for the corresponding $t_{\mathrm{CM}}$ values on the scale of $T_{\mathrm{M}}$. The isofrequency curves obtained from IS measured for LE-EM and EM-LE transitions and already shown in Figures $5 a$ and $5 b$, are at some selected frequencies (of the middle frequency range) presented in Figure 7.

It is obvious that iso-frequency $Z^{\prime}$ and $Z^{\prime \prime}$ values decrease with $T_{M}$ for LE-EM transition (Figure 7a) and increase with $T_{\mathrm{M}}$ for EM-LE transition (Figure $7 \mathrm{~b}$ ), and in both cases, the rate of change decreased over time. Selection of the proper function(s) describing time dependence(s) of iso-frequency impedance values makes 


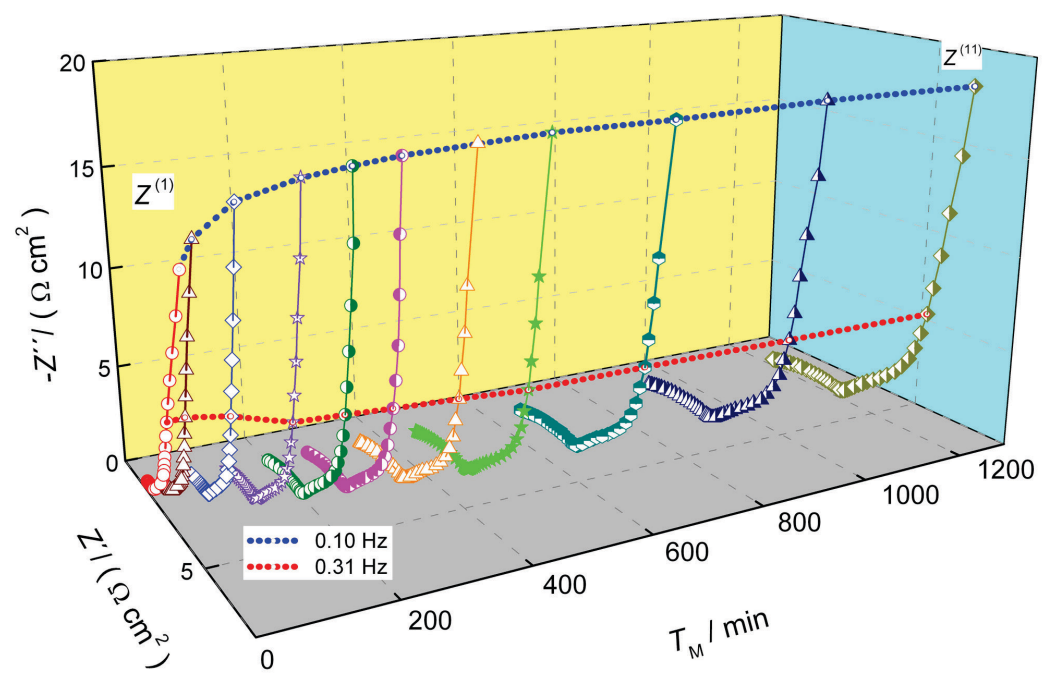

Figure 6. Operating time $\left(T_{M}\right)$ dependence of Nyquist plots taken from Figure $5 \mathrm{~b}$. Iso-frequency points at 0.10 and $0.31 \mathrm{~Hz}$ are connected by dotted lines.

the basis for construction of instantaneous impedance spectra for some chosen time, using the interpolation/extrapolation method. ${ }^{[14,21,22]}$ Several functions, including complex spline ${ }^{[24-26]}$ or combination of two exponential

a
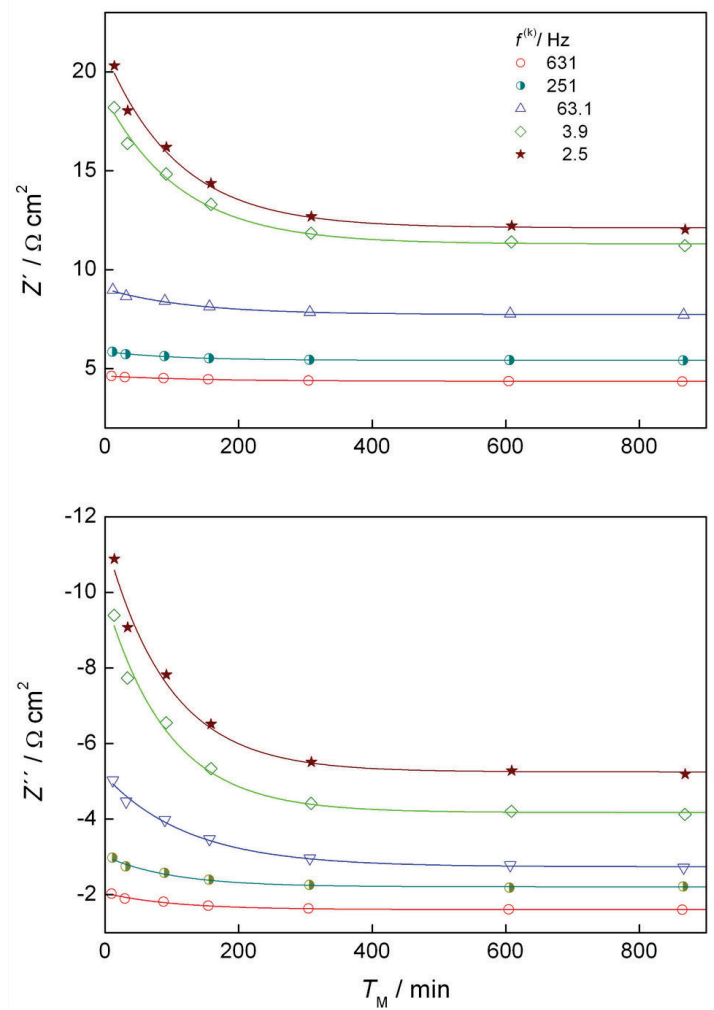

functions, ${ }^{[23]}$ were already used for corrections of nonstationary IS of some conducting polymers.

In the present case of PANI film subjected to redox transitions in both directions of potential change, the

b
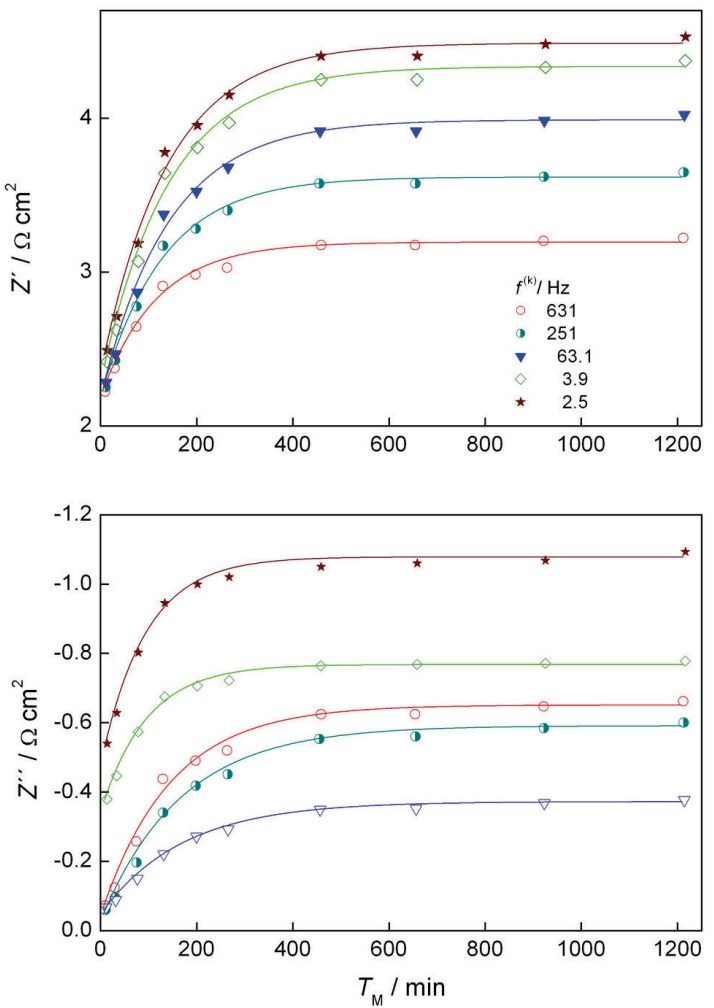

Figure 7. Iso-frequency $Z^{\prime}$ and $Z^{\prime \prime}$ values in dependence on the operating time $\left(T_{M}\right)$ at some selected frequencies for IS taken from: a) Figure 5a; b) Figure 5b. 
simple exponential function, $y(x)=Y_{0}+A e^{x / B}$, was validated by the non-linear curve fitting procedure for all $k=1-60$ isofrequency $Z^{\prime}$ and $Z^{\prime \prime} v s$. $T_{\mathrm{M}}$ dependences. The results of curve fittings are denoted by full lines in Figure 7. Note that $\chi^{2}$ values for all 60 iso-frequency points of each IS ranged from $1 \times 10^{-3}$ to $1 \times 10^{-4}$. The validated exponential function is described by three constants $\left(Y_{0}, A, B\right)$, which for all IS were separately determined for each $k=1-60$ frequency. Now $Z^{\prime}$ and $Z^{\prime \prime}$ values can be numerically calculated at all available (or some selected) $f^{(\mathrm{k})}$ for any chosen time instant on the $T_{\mathrm{M}}$ scale. In such a way, the instantaneous IS $\left(Z_{\text {in }}\right)$ were generated in the form of usual 3-D set $\left(Z^{1}{ }_{\text {in }}{ }^{(k)}, Z^{11}{ }_{\text {in }}{ }^{(k)}\right.$, $\left.f^{(k)}\right)$. Although instantaneous impedance spectra could be calculated not only within, but also out the experimental time domain, ${ }^{[24-26]}$ here, instantaneous IS was for each set of data (already presented in Figures $5 \mathrm{a}$ and $5 \mathrm{~b}$ ), generated by calculations of $Z^{\prime}$ in and $Z^{\prime \prime}$ in values for $\mathrm{k}=1-60$ frequency points at $T_{s}^{(1)}$. In other words, for each series of measurements, the instantaneous IS is defined for the time instant of the beginning of the first IS measurement $\left(Z^{(1)}\right)$.

\section{Measured vs. Instantaneous Impedance Spectra}

In Figure 8 , the calculated $Z_{\text {in }}$ are in usual Nyquist plots compared with the first IS $\left(Z^{(1)}\right)$ measured in the courses of LE-EM and EM-LE transitions ( $c f$. Figures 4 and 5). As stated above, each IS was measured during $t_{\mathrm{m}}=725 \mathrm{~s}$, which is enough time for PANI film to change in both directions of transformation. Instantaneous $Z_{\text {in }}$, however, mimic what would be if IS were measured for all frequencies at the time instant $T_{s},{ }^{[1]}$ taking thus away a time for PANI film change.

Differences between $Z^{(1)}$ and $Z_{\text {in }}$ seen in Figure 8 suggest that generally lower impedances for LE-EM and higher impedances for EM-LE transition would be measured using the conventional EIS technique. In all cases, differences are particularly obvious in semicircle diameters related to charge transfer resistance values. Also, differences could be seen in Warburg parts in cases where they are prominent as $-1 / 2$ slope lines that follow semicircle responses (Figures $8 \mathrm{~b}$ and $\mathrm{c}$ ). The results presented in Figure 8 are in accordance with already given observation that for IS of the overoxidized PEDOT film, the middle frequency range where charge transfer impedance dominates is more affected by time changes than low frequency range where impedance is due to the film pseudo-capacitance. ${ }^{[21,24-26]}$

\section{Impedance Parameter Values Evaluation}

For evaluating impedance parameter values, the general electrical equivalent circuit (EEC) for PANI film electrodes ${ }^{[3]}$ was modified for contributions of experimental artefacts ${ }^{[35,37]}$ and applied in the form presented in Figure 9.

EEC in Figure 9 represents the measured system in terms of impedance due to experimental artefacts, high frequency resistance and impedance of PANI film. Impedance due to experimental artefacts is dominant at the highest frequencies, originated from use of threeelectrode cell and determined by inductive and capacitive parameters $L^{*}$ and $C^{*}{ }^{[35,37]}$ High frequency resistance $\left(R_{\mathrm{HF}}\right)$ is determined mainly by the uncompensated solution resistance, but may also involve the bulk PANI matrix resistance in the case of less conducting PANI. ${ }^{[33,34]}$ PANI film is described in terms of two interfacial (metal/film and film/solution) regions with double-layer capacitive impedances $\left(Z_{\mathrm{C} 1}\right.$ and $\left.Z_{\mathrm{C} 2}\right)$, charge transfer (electrons and ions) resistances $\left(R_{\mathrm{ct} 1}\right.$ and $\left.R_{\mathrm{ct} 2}\right)$, and transmission line a

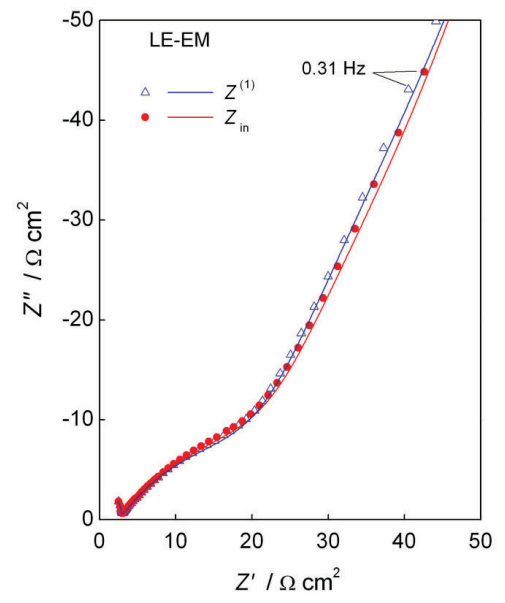

b

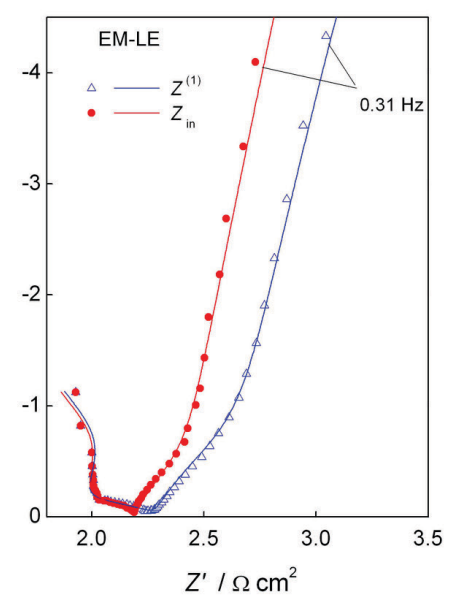

C

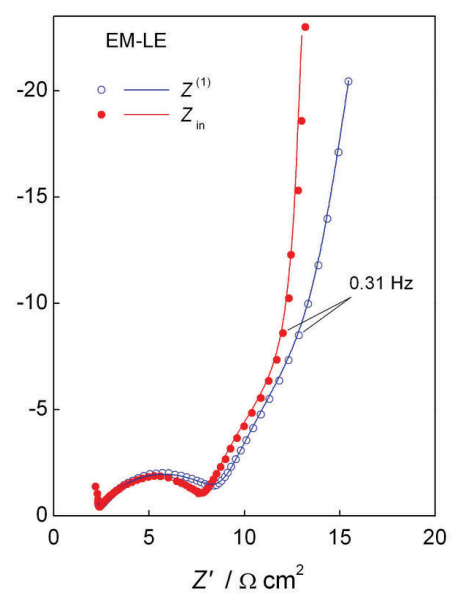

Figure 8. Nyquist plots of $Z^{(1)}$ and $Z_{\text {in }}$ calculated at $T^{(1)}$ for: a) LE-EM transition at $0.05 \mathrm{~V}$; b) EM-LE transition at $0.00 \mathrm{~V}$; c) EM-LE transition at $-0.05 \mathrm{~V}$. 


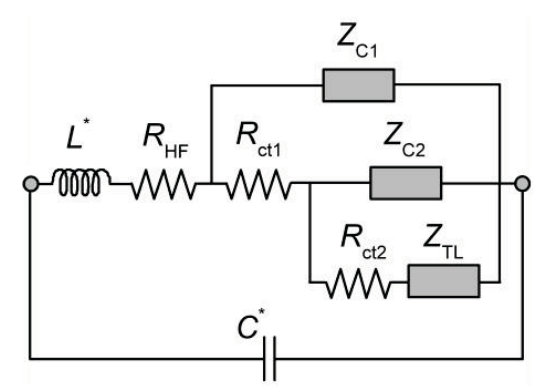

Figure 9. EEC applied for evaluation of impedance parameter values of PANI film.

impedance $\left(Z_{\mathrm{TL}}\right) . Z_{\mathrm{TL}}$ describes the conducting PANI film in terms of diffusion/migration of solution ions in the polymer film and pseudo-capacitance of the inner surface of the opened PANI film. ${ }^{[33,41,42]}$ Impedance/frequency functions of $Z_{\mathrm{C}}$ and $Z_{\mathrm{T} L}$ are defined as follows:

$$
\begin{aligned}
& Z_{c}(i \omega)=Q^{-1}(i \omega)^{-\alpha} \\
& Z_{T L}(i \omega)=R_{W}(i \omega \tau)^{-0.5} \operatorname{coth}(i \omega \tau)^{0.5}
\end{aligned}
$$

The constant phase element concept instead of ideal capacitance was explored by using eq. (1), where capacitive impedance is described by two impedance parameters $(Q$ and $\alpha)^{[1,2]}$ Impedance $Z_{\mathrm{T} L}$ defined by eq. (2) describes charge transport (diffusion or migration) within the layer by the resistance $\left(R_{\mathrm{W}}\right)$ and relaxation time $(\tau)$, where pseudocapacitance of the PANI film interior $\left(C_{\mathrm{f}}\right)$ is defined as $\tau / R_{\mathrm{W}}{ }^{[32-34,40,41]}$

It can generally be expected and has already been mentioned in discussion of Figure 4 that for the insulating LE-PANI form, all charge transfer/transport resistance values are rather high, making double-layer impedance(s) to dominate in IS. The opposite is true for the conducting EMPANI form, where charge transfer/transport resistance values are rather small, making $C_{f}$ related impedance of the open active layer of PANI film to dominate in IS. All is approved with reduced number of impedance parameter values calculated by CNLS fittings of the EEC in Figure 9 to stationary IS measured at -0.20 and $0.45 \mathrm{~V}$ vs. SCE (cf. Figure 4) which are listed in two peripheral columns of Table 1.

Transformation between these two limited cases would occur within the redox transition range of potentials and is possible only when charge transfer/transport resistance $\left(R_{\mathrm{ct} 1}, R_{\mathrm{ct} 2}\right.$ and $\left.R_{\mathrm{W}}\right)$ values are either decreased (during LE-EM redox transition) or increased (during EM-LE redox transition). This suggests that just the reliability of charge transfer/transport resistance values would be crucial for proper characterization of the PANI film state. Since nonstationary impedance spectra are generally measured in the redox transition range, calculations of instantaneous IS could help in obtaining reliable charge transfer/transport resistance values.

Table 1. Impedance parameter values calculated by CNLS fittings of EEC in Figure 9 to IS of PANI film measured at denoted

\begin{tabular}{|c|c|c|c|c|c|c|c|c|}
\hline \multirow[b]{2}{*}{ Parameter } & \multirow{2}{*}{$\frac{\mathrm{LE}}{\mathrm{at}-0.20 \mathrm{~V}}$} & \multicolumn{2}{|c|}{$\begin{array}{c}\text { LE-EM transition } \\
\text { at } 0.05 \mathrm{~V}\end{array}$} & \multicolumn{2}{|c|}{$\begin{array}{c}\text { EM-LE transition } \\
\text { at }-0.05 \mathrm{~V}\end{array}$} & \multicolumn{2}{|c|}{$\begin{array}{c}\text { EM-LE transition } \\
\text { at } 0.00 \mathrm{~V}\end{array}$} & \multirow{2}{*}{$\begin{array}{c}\begin{array}{c}E M \\
\text { at } 0.45 \mathrm{~V}\end{array} \\
Z^{(1)}\end{array}$} \\
\hline & & $Z^{(1)}$ & $Z_{\text {in }}$ at $T_{s}^{(1)}$ & $Z^{(1)}$ & $Z_{\text {in }}$ at $T_{s}^{(1)}$ & $Z^{(1)}$ & $Z_{\text {in }}$ at $T_{s}^{(1)}$ & \\
\hline$R_{\mathrm{HF}} / \alpha \Omega \mathrm{cm}^{2}$ & 2.66 & 2.74 & 2.74 & 2.30 & 2.30 & 1.90 & 1.89 & 1.70 \\
\hline$Q_{1} / \Omega^{-1} s^{\alpha 1} \mathrm{~cm}^{-2}$ & 0.00083 & 0.0039 & 0.0040 & 0.0017 & 0.0023 & $* 0.008$ & $* 0.003$ & - \\
\hline$\alpha_{1}$ & 0.86 & 0.54 & 0.54 & 0.66 & 0.68 & 0.56 & 0.63 & - \\
\hline$R_{\mathrm{ct} 1} / \Omega \mathrm{cm}^{2}$ & $1.6 \times 10^{4}$ & 30 & 33 & 6.74 & 5.99 & 0.40 & 0.29 & - \\
\hline$Q_{2} / \Omega^{-1} S^{\alpha 2} \mathrm{~cm}^{-2}$ & - & 0.0063 & 0.0057 & 0.037 & 0.029 & 0.069 & 0.078 & - \\
\hline$\alpha_{2}$ & - & 0.81 & 0.82 & 0.82 & 0.92 & 0.90 & 0.92 & - \\
\hline$R_{\mathrm{ct} 2} / \Omega \mathrm{cm}^{2}$ & - & - & - & - & - & - & - & - \\
\hline$R_{w} / \Omega \mathrm{cm}^{2}$ & - & *503 & *565 & 52 & 29 & 5.2 & 4.0 & 0.14 \\
\hline$\tau / s$ & - & $* 1.7$ & $* 1.8$ & 1.80 & 1.66 & 0.24 & 0.18 & 0.023 \\
\hline$C_{f} / F_{c m}^{-2}$ & - & 0.00338 & 0.00319 & 0.0346 & 0.0368 & 0.041 & 0.045 & 0.164 \\
\hline$L^{*} \times 10^{6} / \mathrm{H} \mathrm{cm}^{2}$ & 1.61 & 2.82 & 2.82 & 2.09 & 2.08 & 1.81 & 1.79 & 1.5 \\
\hline $\mathrm{C}^{*} \times 10^{7} / \mathrm{Fcm}^{-2}$ & 5.50 & 5.59 & 5.59 & 6.25 & 6.29 & 7.23 & 7.28 & 8 \\
\hline$\chi^{2} \times 10^{4}$ & 8.9 & 5.1 & 4.3 & 1.9 & 4.3 & 3.4 & 3.1 & 8.5 \\
\hline
\end{tabular}
polarization potentials $\left(Z^{(1)}\right)$ and corrected for nonstationarity $\left(Z_{\text {in }}\right)$ 
Values of impedance parameters obtained by CNLS fittings of the EEC in Figure 9 to impedance spectra of $Z^{(1)}$ and $Z_{\text {in }}$ presented in Figure 8 are arrayed according to impedance magnitudes and also listed in Table 1.

Well fitted curves with acceptably low $\chi^{2}$ values suggest the proper choice of the EEC in impedance data analysis of PANI film submitted to redox transitions. In all cases of redox transition, PANI film is described by almost all parameters defined by the EEC of Figure 9 , except $R_{\mathrm{ct} 2}$ value(s) that could not been resolved, probably due to overlapping with $Z_{\mathrm{TL}}$ impedance in all cases. Also, all impedance parameters were evaluated with rsd of few percents (not shown because of saving space), except in some cases where uncertainties were somewhat higher (values denoted by asterisks in Table 1).

In accordance with discussion of Figure 8 , in all cases of nonstationary impedance spectra, certain discrepancy exists between data values obtained for $Z^{(1)}$ and $Z_{\text {in }}$. Differences are indisputable for $R_{\mathrm{ct}}$ and $R_{\mathrm{W}}$ resistance parameters, while much less and almost random differences are noticed for capacitance related $Q_{1}, Q_{2}$ and $C_{\mathrm{f}}$ values calculated as $\tau / R_{\mathrm{w}}$. As expected for parameters not closely related to PANI film, not any difference can be noticed for the values of $R_{\mathrm{HF}}$ and parameters $L^{*}$ and $C^{*}$ due to experimental artefacts. A clear tendency of $R_{\mathrm{ct} 1}$ and $R_{\mathrm{W}}$ resistance values evaluated from $Z_{\text {in }}$ to be higher than measured during LE-EM and lower than measured during EM-LE transitions, pointed to underestimation of these parameter values in the first case and overestimation in the second case, respectively. Whereas overestimation of charge transfer resistance values has already been stressed after comparison of nonstationary and instantaneous IS of PEDOT and POEA polymer films, ${ }^{[21,23-26]}$ possible underestimation of impedance parameter values due to nonstationarity has not been reported so far.

\section{CONCLUSIONS}

Prominent nonstationarities of impedance spectra of PANI film electrodes in $0.05 \mathrm{M} \mathrm{H}_{2} \mathrm{SO}_{4}$ recorded at transition redox potentials can be corrected by using 4-D method of instantaneous impedance spectra calculations. For such a purpose, a number of sequentially measured IS were collected at every polarization potential of interest. For each IS, the resulting data set contained impedance values, specific frequencies of measurements and central time instant of each frequency point measurement. On the basis of strictly defined functions between iso-frequency values of real and imaginary impedance parts and the real operating time of the experiment, impedance values at specific frequencies of instantaneous IS were calculated for the time instant of the beginning of the first IS measurement. Measured and instantaneous impedance spectra were analysed according to the general electrical equivalent circuit already proposed for PANI film, what resulted in a number of impedance parameters values. Comparison between the first measured and instantaneous IS and also between evaluated impedance parameter values indicated that false impedance parameter values, primarily charge transfer and charge transport resistance values, would be obtained if corrections for nonstationarity were not taken into account.

\section{REFERENCES}

[1] E. Barsoukov, J. R. Macdonald, Impedance Spectroscopy Theory, Experiment, and Applications, Second Edition, John Wiley \& Sons, Inc., 2005.

[2] B. Tribollet, M. E. Orazem, Electrochemical Impedance Spectroscopy, John Wiley \& Sons, Inc., 2008.

[3] N. Bonanos, P. Pissis, J. R. Macdonald, Impedance spectroscopy of dielectrics and electronic conductors, in: Characterization of Materials (Ed: E. N. Kaufmann), John Wiley \& Sons, Inc., 2012.

[4] K. Pandey, S. T. A. Islam, T. Happe, F. A. Armstrong, PANAS, 2017, 114, 3843.

[5] F. Jütner, Electrochim. Acta 1990, 35, 1501.

[6] J. P. Christophersen, J. Morrison, W. Morrison, C. Motloch, SAE Int. J. Passeng. Cars Electron. Electr. Syst. 2012, 5(1) 246.

[7] B. Pejčić, R. De Marco, Electrochim. Acta 2006, 51, 6217.

[8] J. Ryl, K. Darowicki, P. Slepski, Corr. Sci. 2011, 53, 1873.

[9] K. Darowicki, J. Kawula, Electrochim. Acta 2004, 49, 4829.

[10] N. Wagner, M. Schulze, Electrochim. Acta 2003, 48, 3899.

[11] S.-M. Park, J.-S. Yoo, B.-Y. Chang, E.-S. Ahn, Pure Appl. Chem. 2006, 78, 1069.

[12] D. Klotz, M. Schönleber, J.P. Schmidt, E. Ivers-Tiffée, Electrochim. Acta 2011, 56, 8763.

[13] Q. Yin, G. H. Kelsal, D. J. Vaughan, N. P. Brandon, J. Electrochem. Soc. 2001, 148 (3), A200.

[14] Z. Stoynov, B. Savova-Stoynov, T. Kossev, J. Power Sources, 1990, 30, 275.

[15] K. Darowicki, J. Electroanal. Chem. 2000, 486, 101.

[16] J.-S. Joo, S.-M. Park, Anal. Chem. 2003, 75, 455A.

[17] T. Breugelmans, J. Lataire, T. Muselle, E. Tourwé, R. Pintelon, A. Hubin, Electrochim. Acta 2012, 76, 375.

[18] R. L. Sacci, D. A. Harington, ECS Transactions 2009, $19,31$.

[19] P. Slepski, K. Darowicki, E. Janicka, G. Lentka, J. Solid State Electrochem. 2012, 16, 3539.

[20] R. L. Sacci, F. Seland, D. A Harrington, Electrochim. Acta, 2014, 131, 13. 
[21] G. G. Láng, D. Zalka, Physiol. Meas. 2018, 39, 028001.

[22] E. Louarroudi, B. Sanchez, Physiol. Meas. 2018, 39, 028002.

[23] V. Horvat-Radošević, K. Kvastek, K. Magdić Košiček, Bulg. Chem. Commun. 2017, 49, 119.

[24] M. Ujvári, D. Zalka, S. Vesztergom, S. Eliseeva, V. Kondratiev, G. G. Láng, Bulg. Chem. Commun. 2017, 49, 106.

[25] D. Zalka, N. Kovács, K. J. Szekeres, M. Ujvári, S. Vesztergom, S. Eliseeva, V. Kondratiev, G. G. Láng, Electrochim. Acta 2017, 247, 321.

[26] D. Zalka, S. Vesztergom, M. Ujvári, G. G. Láng, J. Electrochem. Sci. Eng. 2018, 8, 151.

[27] G. Inzelt, M. Pineri, J. W. Schultze, M. A. Vorotyntsev, Electrochim. Acta 2000, 45, 2403.

[28] T.-H. Le, Y. Kim, H. Yoon, Polymers 2017, 9, 150.

[29] G. Inzelt, J. Electrochem. Sci. Eng. 2018, 8, 3.

[30] E. Cahók, E. Vieil, G. Inzelt, J. Electroanal. Chem. 2000, 482, 168.

[31] W. A. Marmisollé, D. Posadas, M. I. Florit, J. Phys. Chem. B 2008, 112, 1800.

[32] J. F. Rubinson, Y. P. Kayinamura, Chem. Soc. Rev. 2009, 38, 3339.

[33] V. Lvovich, Electrochem. Soc. Interface, 2009, 18, 62.

[34] G. Inzelt, G. G. Láng, Electrochemical impedance spectroscopy (EIS) for polymer characterization, $\mathrm{Ch}$.
3, in: Electropolymerization: concepts, materials and applications, (Eds: S. Cosnier, A. Karyakin), WileyVCH Verlag GmbH \& Co. KGaA,Weinheim, 2010.

[35] V. Horvat-Radošević, K. Kvastek, J. Electroanal. Chem. 2009, 631, 10.

[36] W. A. Marmisollé, M. I. Florit, D. Posadas, J. Electroanal. Chem. 2012, 673, 65.

[37] V. Horvat-Radošević, K. Kvastek, J. Electroanal. Chem. 2006, 591, 217.

[38] H. Dynh, P. Vanysek, V. Birss, J. Electrochem. Soc. 1999, 146, 3324.

[39] R. Paliukaite, C. M. A. Brett, A. P. Monkman, Electrochim. Acta, 2004, 50, 159.

[40] V S.K. Mondal, K.R. Prasad, N. Munichandraiah, Synth. Metals 2005, 148, 275.

[41] V. Horvat-Radošević, K. Kvastek, M. Kraljić-Roković, Electrochim. Acta 2006, 51, 3417.

[42] Z. Mandić, M. Kraljić Roković, T. Pokupčić, Electrochim. Acta 2009, 544, 2941.

[43] H. Li, J. Wang, Q. Chu, Z. Wang, F. Zhang, S. Wang, J. Power Sourc. 2009, 190, 578.

[44] K. Magdić Košiček, K. Kvastek, V. Horvat-Radošević, J. Solid State Electrochem. 2016, 20, 3003.

[45] Z. Wang, K. J. Aoki, J. Chen. X. Zeng, Int. J. Chem. 2018, 10, 25. 